\title{
On Sharkovsky's cycle coexistence ordering
}

\section{Peter E Kloeden}

A theorem of Sharkovsky on the coexistence of cycles for onedimensional difference equations is generalized to a class of difference equations of arbitary dimension. The mappings defining these difference equations are such that the $i$ th component depends only on the first $i$ independent variables.

\section{Introduction}

In 1964 Sharkovsky [4] proved a remarkable theorem on the coexistence of cycles of different periods for one-dimensional difference equations defined in terms of continuous mappings from a compact interval into itself. Recent work on the chaotic behaviour of such difference equations, in particular the "period three implies chaos"result of Li and Yorke [3], has given fresh significance to this theorem: see Kloeden, Deakin, Tirkel [2].

This note considers the generalization of Sharkovsky's Theorem to difference equations of more than one dimension. First a simple example is given of a two-dimensional difference equation for which Sharkovsky's Theorem does not hold. Then attention is restricted to a class of difference equations which includes all of the above one-dimensional difference equations and important higher dimensional difference equations such as the twisted horseshoe difference equation of Guckenheimer, Oster, and Ipaktchi [1], and it is shown that Sharkovsky's Theorem is valid for all difference equations of any dimension which belong to this class.

Received 15 January 1979. 


\section{Preliminaries}

Let $I$ be a compact subset of $\mathbf{R}^{N}$, where $N \geq 1$, and let $f: I \rightarrow I$ be a continuous mapping. Associated with this mapping there is an $N$-dimensional first order difference equation

$$
x^{n+1}=f\left(x^{n}\right)
$$

which for each $x^{0} \in I$ generates an iterative sequence $x^{0}, x^{1}, x^{2}, \ldots$. Such an iterative sequence is said to form a cycle of period $k$ if $x^{0}, x^{1}, \ldots, x^{k-1}$ are all different and $x^{k}=x^{0}$. Note that here $x^{0}, x^{1}, \ldots, x^{k-1}$ are all fixed points of the $k$ th iterate $f^{k}=f \circ f \circ \ldots \circ f(k$ times $)$ of the mapping $f$.

For a fixed but otherwise arbitrary difference equation (2.1) Sharkovsky [4] defined an ordering $\rightarrow$ on the set of natural numbers $\mathbb{N}$ as

$n_{1} \longrightarrow n_{2}$ if the difference equation (2.1) has a cycle of period $n_{2}$ whenever it has a cycle of period $n_{1}$; and he proved the following theorem.

THEOREM (Sharkovsky). For $N=1, I$ a compact interval and $f: I \rightarrow I$ a continuous mapping, the ordering $\longrightarrow$ satisfies $3 \prec 5 \prec 7 \prec 6 \prec 10 \prec 14 \prec \ldots$

$\longrightarrow 3.2^{n} \longrightarrow 5.2^{n} \longrightarrow 7.2^{n} \longrightarrow \ldots \longrightarrow 2^{n} \longrightarrow 2^{n-1} \longrightarrow \ldots \longrightarrow 2 \longrightarrow 1$.

This ordering will in the sequel be called the Sharkovsky ordering.

Sharkovsky's proof of the above theorem involves little more than repeated use of the Intermediate Value Theorem, but is rather long. For a succinct, English language version of this proof, with minor corrections, see Štefan [5].

Now let $I$ be a closed disc in $\mathbf{R}^{2}$ centred on the origin and let $f: I \rightarrow I$ be defined by

$$
f\left(x_{1}, x_{2}\right)=\left(-\frac{1}{2} x_{1}-\frac{\sqrt{3}}{2} x_{2}, \frac{\sqrt{3}}{2} x_{1}-\frac{1}{2} x_{2}\right)
$$


for all $\left(x_{1}, x_{2}\right) \in I$. Then $f: I \rightarrow I$ and $f$ is continuous. Also every $\left(x_{1}, x_{2}\right) \in I \backslash(0,0)$. belongs to a cycle of period three, whereas $(0,0)$ belongs to a cycle of period one for the difference equation (2.1) associated with this mapping $f$. To see this note that using the complex variable $z=x_{1}+i x_{2}$, the mapping $f$ can be written as

$$
f(z)=a \cdot z
$$

where $a=-\frac{1}{2}+i \frac{\sqrt{3}}{2}$ is a complex cubic root of unity. Thus Sharkovsky's Theorem is not in general valid for $N \geq 2$. This example also shows that the "period three implies chaos" result of $L i$ and Yorke [3] is not in general true for difference equations of more than one dimension.

\section{A class of difference equations}

Let $I$ be a compact subset of $\mathbf{R}^{N}$ of the form

$$
I=\prod_{i=1}^{N} I_{i}
$$

where $I_{i}$ is a compact interval for $i=1,2, \ldots, N$ and let $f: I \rightarrow I$ be a continuous mapping of the form

$$
f_{i}\left(x_{1}, x_{2}, \ldots, x_{N}\right)=f_{i}\left(x_{1}, x_{2}, \ldots, x_{i}\right)
$$

for $i=1,2, \ldots, N$, that is to say a mapping for which the $i$ th component $f_{i}$ depends only on the first $i$ independent variables $x_{1}, x_{2}, \ldots, x_{i}$

Conditions (3.1) and (3.2) are satisfied by all one-dimensional difference equations defined in terms of continuous mappings from a compact interval into itself. When $N=2$ they are also satisfied, for example, by the twisted horseshoe difference equation of Guckenheimer, Oster and Ipaktchi [1], for which $f=\left(f_{1}, f_{2}\right)$ is defined on $I=[0,1]^{2}$ by

$$
f_{1}\left(x_{1}\right)= \begin{cases}2 x_{1} & \text { for } 0 \leq x_{1} \leq \frac{1}{2}, \\ 2\left(1-x_{1}\right) & \text { for } \frac{1}{2} \leq x_{1} \leq 1,\end{cases}
$$




$$
f_{2}\left(x_{1}, x_{2}\right)=x_{1} / 2+x_{2} / 10+\frac{3}{2} \text { for all }\left(x_{1}, x_{2}\right) \in I \text {. }
$$

Sharkovsky's Theorem holds for such difference equations.

THEOREM. For any $N \geq 1, I \subset \mathbf{R}^{N}$ a compact set of the form (3.1) and $f: I \rightarrow I$ a continuous mapping of the form (3.2), the ordering $\longrightarrow$ is the Sharkovsky ordering.

To prove this theorem the following lemma is required. In it $N \geq 2$, $\hat{I}=\prod_{i=1}^{N-1} I_{i}, \hat{f}=\left(f_{1}, f_{2}, \ldots, f_{N-1}\right), \hat{x}=\left(x_{1}, x_{2}, \ldots, x_{N-1}\right)$, and

$$
\hat{x}^{n+1}=\hat{f}\left(\hat{x}^{n}\right),
$$

where $I$ is of the form (3.1) and $f: I \rightarrow I$ is a continuous mapping of the form (3.2).

LEMMA. If for any $q=1,2,3, \ldots$ equation (3.3) has a cycle of period $q$, then equation (2.1) also has a cycle of period $q$.

Proof. For a mapping $f$ of the form (3.2) and $x=\left(\hat{x}, x_{N}\right)$, equation (2.1) can be written

$$
\left\{\begin{array}{l}
\hat{x}^{n+1}=\hat{f}\left(\hat{x}^{n}\right) \\
x_{N}^{n+1}=f_{N}\left(\hat{x}^{n}, x_{N}^{n}\right) .
\end{array}\right.
$$

Let $\hat{n}^{0}, \hat{n}^{1}, \ldots, \hat{n}^{q-1}$ be a cycle of period $q$ of equation (3.3), and define a mapping $h: I_{N} \rightarrow I_{N}$ by

$$
h\left(x_{N}\right)=f_{N}\left(\hat{n}^{q-1}, f_{N}\left(\hat{\eta}^{q-2}, \ldots, f_{N}\left(\hat{n}^{0}, x_{N}\right) \ldots\right)\right)
$$

for all $x_{N} \in I_{N}$. Then $h$ is a continuous mapping from the compact interval $I_{N}$ into itself, so it has a fixed point $\eta^{*}=h\left(\eta^{*}\right)$ in $I_{N}$. Define

$$
\eta_{N}^{0}=\eta^{*}, \eta_{N}^{1}=f_{N}\left(\hat{n}^{0}, \eta_{N}^{0}\right), \ldots, \eta_{N}^{q-1}=f_{N}\left(\hat{n}^{q-2}, \hat{\eta}_{N}^{q-2}\right) .
$$

Then $\eta_{N}^{0}=\eta^{*}=h\left(\eta^{*}\right)=f_{N}\left(\hat{n}^{q-1}, \eta_{N}^{q-1}\right)$, so 


$$
n^{0}=\left(\hat{n}^{0}, \eta_{N}^{0}\right), n^{1}=\left(\hat{n}^{1}, n_{N}^{1}\right), \ldots, n^{q-1}=\left(\hat{n}^{q-1}, \eta_{N}^{q-1}\right)
$$

is a cycle of period $q$ of equation (3.4), that is of equation (2.1) with the mapping $f$ of the form (3.2).

Proof of theorem. The theorem is proved by induction on $N$. By Sharkovsky's Theorem it is true when $N=1$. Suppose that $N \geq 2$ and that the ordering $\longrightarrow$ for the $(N-1)$-dimensional difference equation (3.3) is the Sharkovsky ordering. It will be shown that the ordering $<$ for equation (3.4) is then also a Sharkovsky ordering.

Let $\eta^{0}, \eta^{1}, \ldots, n^{p-1}$ be a cycle of equation (3.4) of period $p=(2 k+1) 2^{2}$. Then equation (3.3) has a cycle $\hat{n}^{0}, \hat{n}^{I}, \ldots, \hat{n}^{q-1}$ of period $q$, where $q$ divides $p$, that is $q=(2 j+1) 2^{i}$ where $0 \leq j \leq k, 0 \leq i \leq 2$, and $2 j+1$ divides $2 k+1$. There are two cases to be considered.

Case $1(j=0)$. Here $q=2^{i}$ for some $0 \leq i \leq 2$, so equation (3.3) has a cycle of period $2^{i}$. As $\longrightarrow$ is the Sharkovsky ordering for this difference equation, there are thus cycles of periods

$$
2^{i-1} \longrightarrow \ldots \longrightarrow 2 \longrightarrow 1
$$

for equation (3.3). Hence by the lemma, equation (3.4) also has cycles of periods

$$
2^{i-1} \longrightarrow \ldots \longrightarrow 2 \longrightarrow 1 .
$$

Now let $h: I_{N} \rightarrow I_{N}$ be defined as in (3.5). As $\eta^{0}, \eta^{1}, \ldots, \eta^{p-1}$ is a cycle of period $p$ of equation $(3.4)$ and as $\hat{n}^{0}, \hat{n}^{1}, \ldots, \hat{n}^{q-1}$ is a cycle of period $q=2^{i}$ of equation (3.3), this means that the onedimensional difference equation

$$
x_{N}^{n+1}=h\left(x_{N}^{n}\right)
$$

has a cycle of period $(2 k+1) 2^{z-i}$. Hence by Sharkovsky's Theorem, equation (3.6) has cycles of all periods

$$
(2 k+3)^{2-i} \longrightarrow(2 k+5) 2^{l-i} \longrightarrow \ldots \longrightarrow 2^{n} \longrightarrow \ldots \leftrightarrow 2 \longrightarrow 1
$$


when $k \geq 1$ or

$$
2^{l-i-1}<\ldots<2 \longrightarrow 1
$$

when $k=0$.

Let $x_{N}^{0}, x_{N}^{1}, \ldots, x_{N}^{r-1}$ be a cycle of period $r$ for equation (3.6), and define

$$
\begin{aligned}
\zeta_{N}^{s q} & =x_{N}^{s} \\
\zeta_{N}^{s q+t+1} & =f_{N}\left(\hat{n}^{t}, \zeta_{N}^{s q+t}\right),
\end{aligned}
$$

for $s=0,1, \ldots, r-1$ and $t=0,1, \ldots, q-1$, where $q=2^{i}$. Then

$$
\left(\hat{n}^{0}, \zeta_{N}^{0}\right),\left(\hat{n}^{1}, \zeta_{N}^{1}\right), \ldots,\left(\hat{n}^{q-1}, \zeta_{N}^{q-1}\right),\left(\hat{n}^{0}, \zeta_{N}^{q}\right), \ldots,\left(\hat{n}^{q-1}, \zeta_{N}^{r q-1}\right)
$$

is a cycle of period $r q=r 2^{i}$ of equation (3.4). Doing this for each $r$ for which equation (3.6) has a cycle of period $r$ shows that equation (3.4) has cycles of all periods

$$
(2 k+3) 2^{2} \longrightarrow(2 k+5) 2^{2} \longrightarrow \ldots<2^{i+1} \longrightarrow 2^{i}
$$

when $k \geq 1$, or

$$
2^{i-1} \longrightarrow \ldots \longrightarrow 2^{i+1} \longrightarrow 2^{i} \text {, }
$$

when $k=0$.

However from the first paragraph of this case, equation (3.4) also has cycles of all periods

$$
2^{i-1} \prec \ldots<2 \longrightarrow 1 .
$$

Hence equation ( 3.4 ) has cycles of all periods

$$
(2 k+3) 2^{2} \longrightarrow(2 k+5) 2^{2} \longrightarrow \ldots \longrightarrow 2^{i} \longrightarrow 2^{i-1} \longrightarrow \ldots \longrightarrow 2 \longrightarrow 1
$$

when $k \geq 1$, or

$$
2^{i-1} \longrightarrow \ldots \longrightarrow 2^{i} \longrightarrow 2^{i-1} \longrightarrow \ldots \longrightarrow 2 \longrightarrow 1
$$

when $k=0$.

Case $2(j \geq 1)$. Here $k \geq 1$ must hold, and equation (3.3) has a 
cycle of period $q=(2 j+1) 2^{i}$. As $\longrightarrow$ is the Sharkovsky ordering for the $(N-1)$-dimensional equation (3.3), this difference equation thus has cycles of all periods

$(2 j+3) 2^{i} \longrightarrow \ldots \longrightarrow(2 k+1) 2^{2} \longrightarrow(2 k+3) 2^{2} \longrightarrow \ldots$

$$
\longrightarrow 2^{n} \longrightarrow \ldots \longrightarrow 2 \longrightarrow 1 \text {. }
$$

Hence by the lemma, equation (3.4) also has cycles of all of these periods.

Combining these two cases shows that the ordering $\longrightarrow$ for the $N$-dimensional equation (3.4), or equivalently here (2.1), is the Sharkovsky ordering. This completes the proof of the theorem.

\section{References}

[1] J. Guckenheimer, G. Oster and A. Ipaktchi, "The dynamics of density dependent population models", J. Math. Biol. 4 (1977), 101-147.

[2] Peter Kloeden, Michael A.B. Deakin, A.Z. Tirkel, "A precise definition of chaos", Nature 264 (1976), 295.

[3] Tien-Yien Li and James A. Yorke, "Period three implies chaos", Amer. Math. Monthly 82 (1975), 985-992.

[4] A.H. Шарновсннй [A.N. Sharkovsky], "Сосуществование цинлов непрерыеного преозразования прямой в себя" [Co-existence of the cycles of a continuous mapping of the line into itself], Ukrain. Mat. そ. 16 (1964), 61-71.

[5] P. Stefan, "A theorem of Sarkovskii on the existence of periodic orbits of continuous endomorphisms of the real line", Commun. Math. Phys. 54 (1977), 237-248.

School of Mathematical and Physical Sciences, Murdoch University,

Murdoch,

Western Australia. 